\title{
IdeAs
}

Idées d'Amériques

12 | 2018

Le tourisme dans les Amériques

\section{Une relation en attente d'évolution : les États-Unis et Cuba}

Isabelle Vagnoux

\section{(2) OpenEdition}

1 Journals

Édition électronique

URL : https://journals.openedition.org/ideas/4787

DOI : $10.4000 /$ ideas.4787

ISSN : 1950-5701

Éditeur

Institut des Amériques

Référence électronique

Isabelle Vagnoux, "Une relation en attente d'évolution : les États-Unis et Cuba », IdeAs [En ligne], 12 |

2018, mis en ligne le 21 décembre 2018, consulté le 20 octobre 2022. URL : http://

journals.openedition.org/ideas/4787 ; DOI : https://doi.org/10.4000/ideas.4787

Ce document a été généré automatiquement le 20 octobre 2022

\section{(c) (i) (9)}

Creative Commons - Attribution - Pas d'Utilisation Commerciale - Pas de Modification 4.0 International - CC BY-NC-ND 4.0

https://creativecommons.org/licenses/by-nc-nd/4.0/ 


\title{
Une relation en attente d'évolution : les États-Unis et Cuba
}

\author{
Isabelle Vagnoux
}

\section{RÉFÉRENCE}

Jorge I. Dominguez, Rafael M. Hernandez, Lorena G. Barberia, Debating U.S.-Cuban Relations: How Should We Now Play Ball?, New York, Routledge, 2017, 2 e edition

Francisco López Segrera, The United States and Cuba. From Closest Enemies to Distant Friends, New York, Rowman and Littlefield, 2017

Richard E. Feinberg, Open for Business. Building the New Cuban Economy, Washington, Brookings Institution, 2016

1 Alors que, pour la première fois depuis soixante ans, Cuba n'est plus gouvernée par un Castro - bien que la famille reste très présente aux postes stratégiques, notamment à la tête du parti communiste (Raúl) et des services de contre-espionnage (Alejandro) - ; alors que les relations avec les États-Unis ont subi un brusque coup de froid avec l'arrivée au pouvoir de Donald Trump, l'avenir de Cuba et de sa relation avec les ÉtatsUnis demeure ponctué de points d'interrogation. Nul ne saurait prédire si la phase de transition actuelle entre l'ère castriste et l'ère post-castriste donnera naissance un jour à une relation constructive et apaisée, et dans quel délai. D'autant que les Amériques ne sont pas figées non plus. Le nouveau président cubain, Miguel Díaz-Canel, a pris ses fonctions en avril 2018 dans un contexte hémisphérique qui n'est plus guère favorable au « socialisme » cubain. Outre l'administration Trump à Washington, il doit également faire face à la débâcle du plus solide allié régional de Cuba, le Venezuela, à son corollaire, la perte d'influence du front de gauche (l'Alliance bolivarienne pour les Amériques, ALBA), et au virage très droitier pris par des pays jusqu'alors gouvernés par des partis de gauche ou de centre gauche (Argentine, Brésil, Chili, par exemple). D'où une vaste tournée du président cubain en novembre 2018, dans des contrées plus 
lointaines, comme la Russie, la Chine, la Corée du Nord, le Vietnam et le Laos, que Washington ne voit guère d'un très bon œil.

2 En cette période d'incertitude et d'entre-deux, entre les avancées historiques de 2014janvier 2017 suivies du coup d'arrêt de juin 2017, et de futurs développements encore inconnus, et à la suite du dossier «États-Unis-Cuba : une nouvelle donne? » publié dans le numéro 10 d'IdeAs, Idées d'Amérique en décembre 2017 ( https:// journals.openedition.org/ideas/2095), nous proposons ici un essai bibliographique à partir de trois ouvrages offrant des angles d'approche différents de la relation bilatérale et de l'avenir de Cuba qui permettent de mieux en appréhender la réalité et le détail. Tous ont été publiés pendant la période de «normalisation » historique, en 2016-2017. Les deux premiers, Debating U.S.-Cuban Relations: How Should We Now Play Ball? et The United States and Cuba, From Closest Enemies to Distant Friends, analysent la relation bilatérale. Le troisième, Open for Business. Building the New Cuban Economy, ne traite pas directement de celle-ci mais la dimension économique est susceptible de constituer un axe majeur de la relation entre les deux pays.

Debating U.S.-Cuban Relations: How Should We Now Play Ball ? est la dernière édition, largement revue et mise à jour (il subsiste cependant des passages quelque peu datés qui peuvent surprendre quand on les lit en 2018), d'un ouvrage collectif, publié initialement en 2012 par le Cubain-Américain Jorge I. Domínguez, professeur à Harvard, le Cubain Rafael M. Hernández, rédacteur en chef du journal de sciences sociales Temas, et Lorena G. Barberia, politiste enseignant à l'Université de São Paulo et chercheuse à Harvard. Actualisé au moment de l'embellie entre les deux nations, dans une Amérique latine favorable à Cuba, cet ouvrage n'a pu prendre en compte la déroute du principal allié latino-américain, le Venezuela, et les évolutions politiques en Amérique latine. En d'autres termes, si l'Amérique latine de 2015-2016 a pu faire pression sur Obama pour que Washington sorte de sa politique de guerre froide, l'Amérique latine de 2018-2019 est sans doute moins indulgente envers l'héritage communiste. Publié en janvier 2017, l'ouvrage ne couvre donc pas le refroidissement de l'ère Trump et le détricotage partiel annoncé en juin 2017 de certaines mesures adoptées par l'Administration Obama, notamment en termes de voyages individuels à Cuba et de transactions financières avec des sociétés appartenant aux militaires cubains.

4 L'originalité de cet ouvrage thématique dense repose sur de passionnants regards croisés entre analyses cubaines d'une part et nord-américaines ou européennes d'autre part, sur la relation bilatérale dans son ensemble (chapitres introductifs de Jorge I. Domínguez et Rafael M. Hernández) et sur les thématiques de sécurité, sur le lien avec l'Union européenne, y compris dans le duo cubano-américain, sur l'économie, sur les flux migratoires ou les échanges culturels. Solidement argumentés, ces chapitres présentent tous un rappel historique et une fine analyse des développements récents et les inscrivent dans un cadre international plus large.

5 La relation entre les États-Unis et Cuba, vieille de deux siècles, apparaît comme paradoxale, modelée par l'attraction et la répulsion, la coopération et la confrontation, une défiance réciproque entre "ennemis intimes", selon la formule de Rafael Hernández. En dépit d'une proximité culturelle intense, la relation est animée par des acteurs politiques multiples, souvent prisonniers de leur intransigeance et de leurs préjugés, empêtrés dans une mentalité d' "assiégé " (Cuba) et de "surpuissance " (États-Unis). La nature du régime politique cubain est une obsession pour les deux pays : pour Washington qui voudrait le faire évoluer, pour La Havane qui s'y accroche 
au nom de la souveraineté nationale et de son indépendance. Les deux pays tendent à voir le monde différemment, ce qui les empêche d'avancer sur la voie de relations plus étroites (Hal Klepak, 95) : côté cubain, une solidarité affichée avec les mouvements «progressistes » voire révolutionnaires, alors que Washington n'a jamais fait mystère de sa forte préférence pour la stabilité et le statu quo. Pourtant, les auteurs mettent en relief une coopération réussie, mais sans publicité, sur les questions de terrorisme, de lutte anti-drogue, d'immigration, de catastrophes naturelles.

6 L'Union européenne, premier partenaire commercial de l'île et premier investisseur, s'invite dans la relation États-Unis-Cuba. Le lien transatlantique est renforcé par l'Accord de dialogue politique et de coopération, signé en 2016 et entré en vigueur fin 2017. L'Union européenne comme les États-Unis sont vus comme cherchant tous deux à utiliser la relation bilatérale pour atteindre des objectifs d'influence politique, alors que Cuba l'utilise pour éliminer les restrictions qui freinent son développement (126). En revanche, contrairement à Washington, l'UE ne remet pas en cause les décisions souveraines de Cuba et les nationalisations, par exemple (150). S'il parait exagéré de voir les négociations préalables à l'Accord comme ayant eu une influence quelconque sur Washington, le fait même qu'un verrou ait sauté a pu faciliter le dialogue avec Washington. La relation transatlantique est devenue une sorte d'exemple mais ne saurait se substituer à la relation ancienne et forte avec les États-Unis, si d'aventure la « normalisation » aboutissait à la levée de l'embargo.

7 Au plan économique, Archibald Ritter explore les effets d'une normalisation totale des relations, notamment avec la levée hautement symbolique de l'embargo (stade qui n'était pas atteint lorsqu'Obama a quitté la Maison-Blanche et qui ne semble pas d'actualité à cette heure) et voit Cuba comme principal bénéficiaire. Au contraire, le Cubain Jorge Mario Sanchez Egozcue dissocie la prospérité économique cubaine, fortement liée à la mise en place de réformes structurelles, des heurs et malheurs de la relation bilatérale. Une amélioration des relations économiques serait un " complément » appréciable mais nullement indispensable, et surtout pas au prix de sacrifices sociaux. Tout comme Arturo Valenzuela, ancien membre de l'Administration Obama, l'avait souligné dans un entretien en décembre 2017 (https:// journals.openedition.org/ideas/2230), Sanchez Egozcue met en relief les résistances internes à Cuba et l'inertie qui empêchent la relation de progresser. Il enjoint ses compatriotes d'abandonner la mentalité « de forteresse assiégée » car «ce n'est pas le monde qui s'adaptera à Cuba » (199). Pour que la relation bilatérale soit harmonieuse, Cuba doit protéger sa souveraineté et asseoir sa prospérité. De son côté, Washington ne saurait dicter sa volonté.

8 L'immigration constitue l'un des domaines où le dialogue entre les deux pays a été régulier, en dehors des périodes de crise qui ont ponctué la relation depuis les années 1960. Plusieurs accords ont été signés depuis 1984, et quelque 20000 immigrants cubains sont acceptés chaque année aux États-Unis. Ceux-ci s'ajoutent aux proches parents de ressortissants américains et à la politique dite de "wet foot/dry foot » en vigueur depuis 1995, qui accepte tout Cubain parvenant à atteindre le sol américain, alors que ceux interpellés en mer sont renvoyés à Cuba. Le départ, temporaire ou permanent, est également facilité par la fin de l'autorisation de sortie du territoire cubain à partir de 2013. Publié au moment d'un ultime changement de politique, l'ouvrage n'a pu tenir compte d'un corollaire inattendu de la "normalisation ", instamment demandé par le gouvernement cubain, à savoir la fin du régime 
d'exception et l'alignement de l'immigration cubaine sur le régime général. En d'autres termes, depuis janvier 2017, la politique de tolérance a disparu, les Cubains ne sont plus vus comme des "réfugiés politiques" ou des "exilés", et tout Cubain entrant illégalement sur le sol américain est susceptible d'être renvoyé à Cuba. La situation s'est complexifiée avec la réduction drastique du personnel diplomatique américain (et canadien) à Cuba à la suite des problèmes de santé inexpliqués dont ont souffert les diplomates nord-américains en 2017. Devant la difficulté d'obtenir des visas dans ces conditions, la migration tend à se faire désormais via des pays tiers.

Un regard croisé sur les échanges culturels vient conclure l'ouvrage, soulignant les difficultés pour les étudiants et les chercheurs cubains de venir étudier ou présenter leurs travaux ou bien encore enseigner aux États-Unis, et surtout l'absence de logique dans l'attribution des visas temporaires, empêchant ainsi toute collaboration stable. Côté cubain, les touristes, étudiants, chercheurs, hommes d'affaires américains sont vus par une partie du gouvernement cubain et certains intellectuels comme une sorte de " cinquième colonne » (284), vouée à saper le fondement socialiste de la société cubaine et, à terme, à mettre en péril l'indépendance du pays. En d'autres termes, les échanges " de personne à personne », encouragés par l'administration Obama, ne seraient qu'une nouvelle stratégie pour atteindre un objectif inchangé : le changement de régime. Milagros Martínez Reinosa invite ses compatriotes à voir la relation avec les Américains " sans naïveté mais également avec moins de préjugés » (285).

Cet ouvrage très complet et mesuré constitue une lecture indispensable pour tous ceux qui s'intéressent à cette relation fascinante. Les Cubains-Américains, dont on connaît le rôle moteur, sont peu présents et n'apparaissent vraiment que dans le développement de leur influence dans la politique migratoire états-unienne. Mais il s'agit là sans doute d'un choix diplomatique, les Cubains ayant quelque mal à parler d'eux sereinement. Tous les auteurs parviennent aux mêmes conclusions, mesurées et prudentes: une coopération existante qui ne demande qu'à être étendue, mais la "normalisation» totale ne pourra se faire que très progressivement (Hal Klepak trouve d'ailleurs le terme curieux puisque, selon lui, la relation n'a jamais été «normale», 96). Jorge Domínguez n'exclut pas, pour sa part, un nouveau faux-pas qui empêcherait de remodeler les relations (57). Des nuances sont perceptibles entre auteurs nordaméricains et cubains: les premiers tendent à mettre l'accent sur la nécessité de poursuivre l'ouverture de la société et de l'économie cubaines; les seconds, toujours inquiets de l'asymétrie entre les deux pays, insistent sur la nécessité de respecter l'indépendance, la souveraineté de Cuba et ses choix en matière de régime politique.

11 C'est cette dernière ligne que suit clairement le second ouvrage généraliste de cet essai, The United States and Cuba. From Closest Enemies to Distant Friends. Francisco López Segrera, universitaire cubain et ancien membre de l'Unesco, présente un point de vue strictement cubain, proche des discours officiels. Ce court ouvrage publié en 2017 (130 pages dont seulement 90 pages de texte) et traduit de l'espagnol, ne couvre pas la période au-delà de juillet 2015, au moment stratégique de l'embellie où les relations diplomatiques entre les deux pays sont rétablies. L'intérêt de cet ouvrage ne réside pas dans l'apport de sources nouvelles, ou de témoignages - l'auteur n'a pas participé aux négociations entre les deux pays - , mais bien dans la compréhension du prisme cubain. L'organisation de l'ouvrage en trois chapitres se recoupant partiellement génère parfois des répétitions. 

$\mathrm{XVI}^{\mathrm{e}}$ siècle, Cuba n'est pas vue en tant que telle mais en toujours en tant que pont, lien, «Key to the New World", "Key to the Gulf» (38). López Segrera rappelle les déclarations annexionnistes des dirigeants américains du $\mathrm{XIX}^{\mathrm{e}}$ siècle, notamment la célèbre métaphore du Secrétaire d'État John Quincy Adams. Les États-Unis ont " volé » la victoire de Cuba dans la guerre d'indépendance en 1898 et lui ont aussi volé son indépendance (42). Il reconnaît cependant l'intérêt des élites cubaines pour cette relation «néocoloniale » avec les États-Unis, phénomène désormais mis au jour par les historiens dans d'autres pays d'Amérique latine. Selon une posture marxiste classique, il voit l'action américaine uniquement sous l'angle économique, alors que l'intérêt stratégique est passé sous silence. La relation « néocoloniale » qui s'établit avant 1958 a rendu inévitables, affirme-t-il, une révolution et un rapprochement avec l'Union soviétique (32). En d'autres termes, cette politique américaine s'est avérée contreproductive et, pour sa sauvegarde, Cuba n'a eu d'autre choix que de rallier le camp communiste. Il reproche aux États-Unis d'imaginer Cuba, de projeter la vision qu'ils en ont, leurs désirs de la voir remplir tel ou tel rôle, sans jamais l'avoir vu tel qu'elle est, et sans jamais avoir pris en compte la réalité cubaine. Seul Obama est parvenu à faire évoluer cette perception, même si l'embargo est maintenu, de même que les demandes en matière de droits de l'homme et de démocratie et les aspects les plus durs du «blocus » en application de la loi Helms-Burton. Même si, au fond, le but ultime de Washington, le changement de régime, n'a pas changé. Seule la stratégie a changé.

Des efforts d'amélioration des relations ont bien été entrepris à diverses périodes, mais, là encore, pour López Segrera, la responsabilité de l'échec incombe à Washington qui, obsédé par le changement de régime à Cuba, n'a jamais été en mesure d'entrer dans des négociations plus larges. Ainsi, le messianisme et l'impérialisme américains, véritable fil rouge de cet ouvrage, sont responsables de la situation cubaine. Washington aurait dû accepter la nouvelle réalité de la Révolution, accepter l'indemnisation proposée pour les biens nationalisés, comme l'ont fait nombre de pays européens ou le Canada, accepter le nouveau cadre de la relation bilatérale, accepter une relation entre égaux qui respecte la souveraineté cubaine plutôt que de persister dans l'opposition et le blocage.

14 López Segrera montre ensuite comment le pays, dès 1960, fait tout pour ne pas être isolé sur la scène internationale. Cuba serait devenue un exemple pour tous les pays luttant contre la dépendance et le sous-développement. Alors qu'on aurait pu craindre un isolement de l'île après l'effondrement de l'URSS, l'auteur détaille au contraire ses bonnes relations avec de nombreux pays (33-37), en dépit du maintien de la révolution et des entraves états-uniennes, en contrepoint de l'échec de la politique de Washington. Cuba serait ainsi sauvée par le monde multipolaire qui dilue la puissance américaine, lui ouvre des alliances nouvelles (Union européenne, Chine, Russie, Brésil) et une forme de soft power (par l'aide médicale, par exemple), ou bien encore favorise les changements à l'intérieur même de l'île. De nouvelles configurations, favorables à Cuba, se mettent en place en Amérique latine au XxI siècle (Venezuela, Nicaragua, Equateur, Bolivie, Brésil, Argentine, Uruguay, Salvador), mais le récent tournant droitier rend déjà obsolète cette affirmation. Moins messianique, la politique étrangère cubaine se fait plus réaliste, plus discrète. Cuba participe à de multiples organisations internationales ou régionales, malgré son exclusion de l'OEA. À une écrasante majorité, 
les membres de l'ONU soutiennent la levée de l'embargo, d'où le fait que la politique américaine a davantage isolé les États-Unis que Cuba (36).

Le dernier chapitre conte le déroulement du processus historique de "normalisation ", «victoire majeure pour Cuba » (61) qui y gagne bien davantage que les États-Unis, et rend hommage aux principaux acteurs ayant influencé la politique américaine envers Cuba: le Vatican, le Canada, les institutions régionales des Amériques (UNASUR, OEA). S'il énumère les raisons qui ont amené Washington à abandonner son attitude «négative », fort peu de chose sont dites sur celles qui ont poussé Cuba à faire un pas vers Washington. Il ne reste désormais plus à Washington qu'à lever l'embargo et rendre Guantanamo, mais rien n'est dit sur ce que Cuba devrait faire, en termes de droits de l'homme, par exemple.

L'auteur s'enorgueillit qu'aucune politique américaine, même la plus hostile, n'ait pu déstabiliser Cuba et le régime communiste. Le modèle à suivre est clairement le modèle vietnamien ou chinois, avec une économie ouverte mais un régime communiste. Demander à Cuba de s'aligner sur les démocraties occidentales et ne pas le demander à la Chine ou au Vietnam relève d'un deux poids deux mesures inacceptable (68). L'auteur engage son pays à ne jamais renoncer à « l'essence de la nation » (70).

Dans Open for Business: Building the New Cuban Economy, Richard Feinberg, éminent spécialiste de l'Amérique latine, fin connaisseur de Cuba, ancien conseiller de Bill Clinton et membre du think tank Brookings Institution, analyse les perspectives cubaines sous l'angle économique, empruntant à la zone de développement économique de Mariel le slogan «Open for Business ». L'ouvrage s'est construit à partir de plusieurs rapports rédigés pour la Brookings à partir de 2011, s'enrichissant au fil des années et de l'actualité, notamment au moment où, alors qu'il se trouve à Cuba, l'auteur apprend l'annonce soudaine du rétablissement des relations diplomatiques, le 17 décembre 2014 (17 D) et vit en direct l'immense espoir ainsi suscité. L'originalité de cet ouvrage est quadruple. Il se fonde sur un large éventail d'entretiens avec de jeunes Cubains, d'acteurs ou d'experts de la scène économique. En d'autres termes, même rédigé par un expert états-unien, cet ouvrage fait la part belle aux témoignages cubains. L'auteur est parvenu à convaincre les cercles décisionnaires à Washington, obsédés par la dimension politique, que l'économie doit devenir prioritaire. D'autres ouvrages collectifs sur cette question seront publiés depuis ${ }^{1}$. Il trace une véritable feuille de route pour l'éclosion du potentiel cubain. Enfin, il prend le risque d'élaborer trois scénarios à l'horizon 2030, du plus pessimiste au plus optimiste.

Open for Business se place résolument dans l'actualité et dans l'avenir et fait une moindre place au passé que les autres ouvrages de cet essai. Il retrace cependant les efforts cubains pour reconstruire l'économie après la disparition de l'aide soviétique. Les réformes, notamment l'ouverture au tourisme international pour obtenir des devises et la légalisation de l'emploi privé, ont commencé dès les années 1990, pour survivre et non par ouverture idéologique. Mais à partir de 1999, l'idéologie reprend le dessus, le gouvernement revient sur l'ouverture à l'investissement étranger direct et préfère se tourner vers des projets étatiques avec le Venezuela, la Chine ou le Brésil. C'est Raúl Castro, à partir de 2006, qui remet en marche le train des réformes, même si peu de mesures sont prises pour obtenir des résultats. Feinberg explore les succès de petites entreprises dans ce nouvel environnement et suggère des pistes pour une expansion économique future. 
19 Revenant sur l'origine du tournant historique de décembre 2014, Feinberg note un alignement favorable de planètes tel qu'il en existe pour tous les tournants de l'histoire: le rôle de la diplomatie internationale, notamment vaticane et latinoaméricaine qui, en exigeant la participation de Cuba au sommet des Amériques de 2015, a contraint Washington à prendre l'initiative ; les changements en cours à Cuba, que les difficultés économiques poussent à rechercher une relation apaisée avec les États-Unis, non pour changer de régime mais au contraire pour lui permettre de survivre; des partenaires émergents instables (Venezuela); de bonnes relations entre les deux chefs d'État; des changements au sein même de la politique intérieure américaine et une majorité de sondés favorables à une normalisation des relations. Feinberg, fin connaisseur du fonctionnement de la Maison-Blanche, analyse ainsi son raisonnement : en réduisant les tensions bilatérales, la main tendue pourrait amener Cuba à faire évoluer sa politique intérieure vers plus d'ouverture. En d'autres termes, ne pas exiger de concessions ou de changements comme pré-condition à toute négociation, mais les attendre comme conséquence espérée de la normalisation.

Cuba se voit comme un exemple de transition d'un modèle économique étatique à un système plus orienté vers le marché, à l'instar du Vietnam, de la Chine ou des pays d'Europe de l'Est. Les réformes en cours les plus prometteuses pour l'avenir de l'île sont la croissance du secteur des petites entreprises privées; la promesse d'ouverture à l'investissement étranger ; l'émergence de marchés pour les résidences familiales et les voitures; une plus grande liberté pour voyager à l'étranger, depuis 2013. Les études de cas montrent que des joint ventures à succès ont déjà contribué à l'économie cubaine. $\mathrm{Si}$ Cuba améliore son climat d'affaires, l'investissement étranger pourrait jouer un rôle moteur dans la croissance de l'économie.

21 Mais de nombreux problèmes structurels n'ont pas été traités et la croissance économique demeure faible, la production industrielle et agricole ne décolle pas, l'épargne et l'investissement stagnent à des niveaux trop bas pour pousser l'économie vers l'avant. Les exportations de marchandises sont insuffisantes. Dans l'ensemble, les performances économiques ne permettent pas d'élever le niveau de vie de la population. Les salaires sont ridiculement bas et poussent les Cubains à émigrer ou bien à se tourner massivement vers les emplois offrant un accès privilégié aux devises étrangères (tourisme), déformant ainsi le développement économique en gonflant le secteur des services qui représente plus de $80 \%$ de la production nationale.

Feinberg regrette la lenteur du gouvernement cubain à réagir aux propositions américaines, la permanence des obstacles administratifs, juridiques et idéologiques, la réticence de certains membres du gouvernement cubain à l'idée de faciliter le secteur privé, et la volonté de maintenir le contrôle étatique. Si quelques entreprises américaines, notamment dans les nouvelles technologies, ont pu plus facilement obtenir le consentement cubain (Airbnb, Verizon et Sprint, Sony Music), l'embargo entrave les possibilités d'expansion des investisseurs américains. Ce handicap s'ajoute à la volonté cubaine de limiter les échanges et les investissements américains afin de diversifier les partenaires (Europe, Canada, Venezuela, Brésil, Mexique, et de plus en plus Chine, Russie) et ne pas dépendre exclusivement des États-Unis; en d'autres termes, tout faire pour ne pas revenir à la période pré-révolutionnaire. Mais le manque d'efficacité et les blocages administratifs découragent tous les partenaires et la Chine ne fait pas exception. Il est clair que, en filigrane, Feinberg voit le partenaire étatsunien comme le meilleur possible pour Cuba. 
L'ouvrage se conclut sur l'analyse de trois scénarios à l'horizon 2030, "l'inertie, l'affaiblissement, l'embellie », dont les tendances sont déjà perceptibles, ainsi que les réactions américaines envisageables. Dans le premier cas, une sorte de statu quo, la réforme économique avance trop lentement, l'économie stagne, rien ne change au niveau institutionnel, la réforme politique n'avance pas, le peuple est déçu et poursuit ses rêves à l'étranger. Également déçus, les décideurs américains pourraient adopter une ligne plus dure. La perspective de la levée de l'embargo s'éloigne. La deuxième hypothèse ajoute à la première des incohérences politiques et le déclin des institutions, en bref, un scénario vénézuélien. Les États-Unis se détourneraient d'une nation «infernale», selon la formule de Theodore Roosevelt, et l'intervention militaire ne serait pas à exclure. Enfin, le meilleur scénario, celui d'une réussite du modèle hybride État-marché à la vietnamienne, impliquant une bonne croissance économique, une réforme réussie au niveau économique et politique, le renforcement des institutions, une population optimiste qui va de l'avant. Convaincus, les États-Unis lèveraient l'embargo et donc les restrictions sur les investissements et les voyages, et surtout ouvriraient leur immense marché aux produits cubains. Confiant dans la force et l'inventivité du peuple cubain, comme en témoignent les portraits qu'il dresse de jeunes entrepreneurs cubains, Feinberg conclut sur une note raisonnablement optimiste quant à la concrétisation de ce scénario.

De ces trois ouvrages ressortent quelques éléments communs : l'ancienneté et donc la complexité de la relation entre les deux pays, le (trop) lourd poids de la politique intérieure, de chaque côté du détroit, qui hypothèque toute programmation à moyen ou long terme, le formidable potentiel de l'île et de ses habitants, un optimisme raisonnable pour l'avenir. Certains auteurs sont plus nuancés que d'autres, mais il ressort également que les Américains ne voient le salut de Cuba et, partant, de la relation bilatérale, que dans l'ouverture et la réforme, alors que les Cubains insistent sur le respect de «l'essence de la nation» et de ses fondements culturels. Richard Feinberg a prudemment choisi 2030, dans une dizaine d'années, pour que l'un de ses scénarios se concrétise. L'histoire connaît parfois des revirements soudains qui changent la donne du jour au lendemain, mais tous les experts s'accordent aujourd'hui à penser que la «normalisation » pleine et entière de la relation bilatérale prendra du temps.

\section{NOTES}

1. Jorge I. Domínguez (dir.), The Cuban Economy in a New Era: An Agenda for Change Toward Durable Development, Cambridge, Mass., Harvard University Press, 2017 


\section{AUTEURS}

\section{ISABELLE VAGNOUX}

Aix Marseille Université, LERMA, Aix-en-Provence, France

Rédactrice-en-chef d'IdeAs, Idées d'Amérique, Isabelle Vagnoux est professeur des Universités à Aix-Marseille, spécialiste de politique étrangère américaine et des relations avec l'Amérique latine. Elle co-dirige à Aix l'Observatoire des relations extérieures du monde anglophone (OREMA) au sein du LERMA (EA 853). isabelle.vagnoux@univ-amu.fr 